\title{
Electron Mass Operator in a Strong Magnetic Field and Dynamical Chiral Symmetry Breaking
}

\author{
A. V. Kuznetsov円 and N. V. Mikheev' \\ Division of Theoretical Physics, Department of Physics, \\ Yaroslavl State University, Sovietskaya 14, \\ 150000 Yaroslavl, Russian Federation
}

(Dated: April 2, 2002)

\begin{abstract}
The electron mass operator in a strong magnetic field is calculated. The contribution of higher Landau levels of virtual electrons, along with the ground Landau level, is shown to be essential in the leading log approximation. The effect of the electron dynamical mass generation by a magnetic field is investigated. In a model with $N$ charged fermions, it is shown that some critical number $N_{c r}$ exists for any value of the electromagnetic coupling constant $\alpha$, such that the fermion dynamical mass is generated with a doublet splitting for $N<N_{c r}$, and the dynamical mass does not arise at all for $N>N_{c r}$, thus leaving the chiral symmetry unbroken.

PACS numbers: 11.30.Rd, 11.30.Qc, 12.20.Ds
\end{abstract}

Asymptotic properties of the QED diagrams and operators in strong magnetic fields $B \gg B_{e}, B_{e}=m_{e}^{2} / e \simeq$ $4.41 \cdot 10^{13} \mathrm{G}$ ( $e$ is the elementary charge) are of conceptual interest both from the standpoint of the searches of the perturbation theory applicability borders, and also in view of possible applications in astrophysics and in cosmology of the early Universe. The investigations of this type are being performed by many authors during a rather long time. For example, a history of calculations of the electron mass operator in a strong magnetic field lasts more than 30 years already. However, as we show in this Letter, it is too early to put the final point in the problem.

One-loop contribution into the electron mass operator in a strong magnetic field was obtained for the first time by Jancovici [1] in the leading (double) log approximation. Later on, in the papers by Loskutov and Skobelev [2, 3] the attempts were performed to calculate the two-loop contribution and to summarize all the manyloop contributions in the same approximation. A correct formula for the electron mass operator in this approximation was obtained recently in the paper $\llbracket \llbracket$. However, the double log approximation becomes invalid in asymptotically strong magnetic fields [5], because of the crucial influence of the strong magnetic field on the virtual photon polarization operator. This influence provides an appearance of the effective photon mass, $m_{\gamma}^{2}=(2 \alpha / \pi) e B$, which replaces the electron mass in one of the two logarithms. A correct expression for the electron mass operator in the leading (single) log approximation was obtained recently in our paper [6] by the summation of the rainbow Feynman diagrams, in the form:

$$
M=\frac{m_{0}}{1-\left(\alpha_{R} / 2 \pi\right)\left[\ln \left(\pi / \alpha_{R}\right)-\gamma_{\mathrm{E}}\right] \ln \left(e B / m_{0}^{2}\right)}
$$

where $m_{0}$ is the electron mass without field, $\gamma_{\mathrm{E}}=$ $0.577 \ldots$ is the Euler constant, $\alpha_{R}$ is the electromagnetic coupling constant renormalized by the field

$$
\alpha_{R}=\frac{\alpha}{1-(\alpha / 3 \pi) \ln \left(e B / m_{0}^{2}\right)} .
$$

However, the formula (11) has a restricted area of applicability with respect to the parameter $\eta_{0}=\frac{\alpha}{2 \pi} \ln \frac{e B}{m_{0}^{2}}$, because the mass operator (11) tends to infinity at some finite value of this parameter. On the one hand, as it was mentioned in [- $\mid$, this could be the signal of a new physics, the spontaneous breaking of chiral symmetry. On the other hand, it indicates that the formula (1) is not self-consistent at the field values for which $\eta_{0} \sim 1$. It means that the transitions to the nonperturbative regime over the parameter $\eta_{0}$ is necessary. In terms of the diagram technique it leads to the necessity to consider, along with the rainbow diagrams giving the leading log contributions, also the diagrams of a next-to-leading order. As the analysis shows, this extension reduces to a substitution of the electron mass operator instead of mass into the diagrams. It is more convenient, however, to use the Schwinger-Dyson equations as it was performed in Refs. [7, 8, 9, 10].

In this Letter, we realize this program by calculating the electron mass operator to be valid in any asymptotically strong magnetic fields. To obtain a full system of the Schwinger-Dyson equations in the presence of external magnetic field, which corresponds to the irreducible diagrams depicted in Figs. 11 and 2, one should know the exact vertex $\Gamma_{\mu}$ which contains, as is well-known, an infinite number of irreducible diagrams.

Fortunately, the problem is simplified essentially in the strong field limit, as it was shown for the first time in Ref. [11], see also [7, 8]. Namely, the exact vertex $\Gamma_{\mu}$ can be reduced to the bare vertex $\gamma_{\mu}$ in an appropriate gauge.

In the strong field limit, the main contribution into the electron mass operator originates from virtual electrons occupying the lowest Landau level [12]. In this case the 


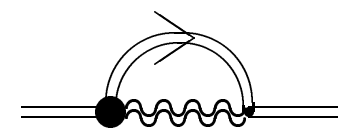

FIG. 1: Feynman diagram for the field-induced contribution into the electron mass operator. Double lines correspond to exact solutions and exact propagators of electrons and photons in an external magnetic field. Bold circle depicts the exact vertex.

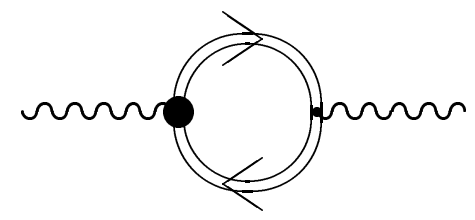

FIG. 2: Feynman diagram for the field-induced contribution into the photon polarization operator.

Fourier transform of the translational invariant part of the exact electron propagator in a magnetic field can be presented in the form

$$
G_{0}^{(e)}(k)=i \exp \left(-\frac{k_{\perp}^{2}}{2 e B}\right)\left[\hat{k}_{\|}-\hat{M}\left(k_{\|}\right)\right]^{-1} O^{(-)},
$$

where $\hat{k}_{\|}=k^{0} \gamma^{0}-k^{3} \gamma^{3}$ (the field is directed along the 3d axis), $\hat{M}\left(k_{\|}\right)$is the sought mass operator, $O^{(-)}=$ $\left(1-i \gamma^{1} \gamma^{2}\right) / 2$ is the projecting operator corresponding to the electron state with the spin antiparallel to the external field direction.

On the other hand, photons of the only one mode ('transversal' in the Adler's notation [13) interact with electrons occupying the lowest Landau level. In this case, in the gauge where $\Gamma_{\mu} \simeq \gamma_{\mu}$ the exact photon propagator can be presented in the form [5]

$$
\begin{aligned}
& G_{\mu \nu}^{(\gamma)}(q)=-i \mathcal{D}\left(q_{\perp}^{2}, q_{\|}^{2}\right) \tilde{\Lambda}_{\mu \nu} \\
& \mathcal{D}\left(q_{\perp}^{2}, q_{\|}^{2}\right)=\frac{1}{q^{2}-\mathcal{P}\left(q_{\perp}^{2}, q_{\|}^{2}\right)} .
\end{aligned}
$$

Here $q_{\perp}^{2}=q_{\mu} \Lambda_{\mu \nu} q_{\nu}, q_{\|}^{2}=q_{\mu} \tilde{\Lambda}_{\mu \nu} q_{\nu}, q^{2}=q_{\|}^{2}-q_{\perp}^{2}$, $\Lambda_{\mu \nu}=\varphi_{\mu \rho} \varphi_{\rho \nu}, \tilde{\Lambda}_{\mu \nu}=\tilde{\varphi}_{\mu \rho} \tilde{\varphi}_{\rho \nu}, \varphi_{\alpha \beta}$ is the dimensionless tensor of external magnetic field, $\varphi_{\alpha \beta}=F_{\alpha \beta} / \sqrt{F_{\mu \nu}^{2} / 2}$, $\tilde{\varphi}_{\alpha \beta}=\frac{1}{2} \varepsilon_{\alpha \beta \rho \sigma} \varphi_{\rho \sigma}$ is the dual tensor. The function $\mathcal{P}\left(q_{\perp}^{2}, q_{\|}^{2}\right)$ is the eigenvalue of the photon polarization operator $\mathcal{P}_{\mu \nu}(q)$, which is depicted by the Feynman diagram Fig. 2. With the reduction $\Gamma_{\mu}=\gamma_{\mu}$, the operator has the form

$$
\begin{aligned}
\mathcal{P}_{\mu \nu}(q) & =-i \frac{\alpha}{4 \pi^{3}} \int d^{4} k \operatorname{Tr}\left[\gamma_{\mu} G^{(e)}(k) \gamma_{\nu} G^{(e)}(k-q)\right] \\
& =\left(\tilde{\Lambda}_{\mu \nu}-\frac{q_{\mu \|} q_{\nu \|}}{q_{\|}^{2}}\right) \mathcal{P}\left(q_{\perp}^{2}, q_{\|}^{2}\right)+\ldots
\end{aligned}
$$

where dots denote the contribution of the other photon modes. Thus the polarization operator (6) is reduced in fact to the one-loop operator.

The mass operator $\hat{M}\left(p_{\|}\right)$corresponding to the irreducible diagram depicted in Fig. 1, in the gauge $\Gamma_{\mu} \simeq \gamma_{\mu}$ and in terms of Eqs. (3) and (4) is reduced to a function $M\left(p_{\|}^{2}\right)$, see Refs. 7] 8]. The following integral equation arises for this function

$$
M\left(p_{\|}^{2}\right)=m_{0}-i \frac{\alpha}{2 \pi^{3}} \int d^{4} k \exp \left(-\frac{k_{\perp}^{2}}{2 e B}\right) \frac{M\left(k_{\|}^{2}\right)}{k_{\|}^{2}-\left[M\left(k_{\|}^{2}\right)\right]^{2}} \frac{1}{(k-p)_{\|}^{2}-k_{\perp}^{2}-\mathcal{P}\left(k_{\perp}^{2},(k-p)_{\|}^{2}\right)}
$$

Integral in the expression (7) does not contain an ultraviolet divergency, because the integration over the momenta $k_{\perp}$ transversal to the field direction has the cutoff $k_{\perp} \sim \sqrt{e B}$.

On the other hand, the photon polarization operator (6), in general, does contain the ultraviolet divergency. As a result, virtual electrons occupying both the lowest and higher Landau levels contribute to the integral. This fact was not taken into account in all previous publications in the field. However, as will be shown below, it leads to very interesting physical consequences.

The function $\mathcal{P}\left(q_{\perp}^{2}, q_{\|}^{2}\right)$ in a strong magnetic field in the one-loop approximation can be extracted, for example, from Ref. 14, see also 15, where the sought function $M\left(q_{\|}^{2}\right)$ should be inserted instead of the field-free mass $m_{0}$ :

$$
\begin{aligned}
\mathcal{P}\left(q_{\perp}^{2}, q_{\|}^{2}\right) & =-\frac{2 \alpha}{\pi} e B \exp \left(-\frac{q_{\perp}^{2}}{2 e B}\right) H\left(\frac{q_{\|}^{2}}{4\left[M\left(q_{\|}^{2}\right)\right]^{2}}\right) \\
& +\frac{\alpha}{3 \pi} q^{2} \ln \frac{e B}{\left[M\left(q_{\|}^{2}\right)\right]^{2}}
\end{aligned}
$$

Here the first term is caused by virtual electrons occupying the lowest Landau level, while the second term contains the contribution from higher Landau levels. The 
function $H(z)$ has the form

$$
H(z)=\frac{1}{2 \sqrt{-z(1-z)}} \ln \frac{\sqrt{1-z}+\sqrt{-z}}{\sqrt{1-z}-\sqrt{-z}}-1 .
$$

In the analysis of Eq. (7), we are interested in the region of parameters $q_{\|}^{2}<0,\left|q_{\|}^{2}\right| \gg\left[M\left(q_{\|}^{2}\right)\right]^{2}$. For large negative values of the argument, the function $H(z)$ is simplified, $H(z) \simeq-1$. The first term in Eq. (8) acquires in this case the meaning of the photon mass squared, $m_{\gamma}^{2}=$ $(2 \alpha / \pi) e B$, induced by a magnetic field. As for the second term in Eq. (8), containing the contribution from higher Landau levels into the photon polarization operator, its role is in renormalization of the electromagnetic constant $\alpha$ in a magnetic field, $\alpha \rightarrow \alpha_{R}$. The expression for the renormalized constant can be obtained from Eq. (2) by the replacement $m_{0} \rightarrow M\left(q_{\|}^{2}\right)$.

Turning back to Eq. (7), it should be mentioned that the integral in the right-hand side exactly corresponds to the one-loop field-induced correction to the electron mass, with the replacement of the field-free mass $m_{0}$ by the sought mass operator $M\left(k_{\|}^{2}\right)$ in the integrand. In the superstrong field limit, the main contribution into the integral (7) in the form of a big logarithm $\ln \left(e B / M^{2}\right)$ originates from the region $\left[M\left(k_{\|}^{2}\right)\right]^{2} \ll\left|k_{\|}^{2}\right| \ll m_{\gamma}^{2} \sim$ $\alpha e B, m_{\gamma}^{2} \lesssim k_{\perp}^{2} \ll e B$. In view of this, the calculation of the integral in Eq. (7) with logarithmic accuracy gives the following result for the mass operator $M\left(p_{\|}^{2}\right)$ in the Euclidean region $p_{\|}^{2}<0,\left|p_{\|}^{2}\right| \ll m_{\gamma}^{2}$ :

$$
M\left(p_{\|}^{2}\right) \simeq M(0)\left[1+\frac{p_{\|}^{2}}{4 e B} \ln \frac{2 e B}{[M(0)]^{2}}\right] .
$$

The expression (10) shows that the electron physical mass which is defined, strictly speaking, as the solution of the dispersion equation $m=M\left(-m^{2}\right)$, can be taken with a great accuracy in the zero point, $m \simeq M(0)$.

In the leading log approximation, we have obtained the following transcendental equation for the electron physical mass from the integral equation (8):

$$
m=m_{0}+m \frac{\alpha_{R}}{2 \pi}\left(\ln \frac{\pi}{\alpha_{R}}-\gamma_{\mathrm{E}}\right) \ln \frac{e B}{m^{2}},
$$

where $\alpha_{R}$ is taken in the point $q_{\|}^{2}=0$, i.e. obtained from Eq. (2) by the replacement $m_{0} \rightarrow M(0)=m$. It is interesting to note that the formula (11) reproduces exactly our result (1), with the substitution of the electron physical mass $m$ instead of the field-free mass $m_{0}$ under the logarithms. The equation (11) solves the problem of finding the electron physical mass for any large values of the magnetic field. It is free of a singularity, unlike the Eq. (1).

An analysis of Eq. (11) shows that its solution in asymptotically strong fields when $m \gg m_{0}$, becomes independent on $m_{0}$ and is reduced in fact to the solution at $m_{0}=0$. This would mean the generation of the dynamical mass of the initially massless electron in a magnetic field. This effect which is also called the dynamical chiral symmetry breaking, was studied in refs. [4, 4, 8, 9, 10, however, the contribution from higher Landau levels into the photon polarization operator was not considered there.

Let us show, that this contribution changes the behaviour of the dynamical mass essentially. Let us extend our analysis to a model with $N$ initially massless fermions $\left(m_{0}=0\right)$ with equal charges $e$ (in a case of different fermion charges $Q_{f} e$, the parameter $N$ has the meaning of $\sum_{f} Q_{f}^{2}$ ). In this case the photon polarization operator $(8)$ is the sum over all fermion loops, i.e. it should be multiplied by $N$. The transcendental equation for the fermion dynamical mass $(m \neq 0)$ can be obtained from Eq. (11) as follows

$$
\frac{\alpha_{R}}{2 \pi}\left(\ln \frac{\pi}{N \alpha_{R}}-\gamma_{\mathrm{E}}\right) \ln \frac{e B}{m^{2}}=1,
$$

where

$$
\alpha_{R}=\frac{\alpha}{1-(N \alpha / 3 \pi) \ln \left(e B / m^{2}\right)} .
$$

The expression (12) allows to reproduce the result of Refs. [7, 8], if the actual dependence of the coupling constant $\alpha_{R}$ on the ratio $e B / \mathrm{m}^{2}$ is formally ignored and $\alpha_{R}=\alpha$ is taken in it. It is remarkable that the constant $C_{1}$ obtained there by a numerical calculation as $C_{1} \simeq 1.82 \pm 0.06$, appears to be $C_{1}=\pi \exp \left(-\gamma_{\mathrm{E}}\right)=$ $1.763877 \ldots$

In Fig. 3 the behaviour of the fermion dynamical mass $m$ divided by $\sqrt{e B}$ is shown versus the number of fermions $N$ and the field-free coupling constant $\alpha$, considered as free parameters of the model.

The dependence is seen to differ essentially from the results of Refs. 7, 8]. Namely, for any value of the coupling constant $\alpha$, such a critical number of fermions $N_{c r}$ exists that for $N<N_{c r}$ two values of the fermion dynamical mass are generated. For $N>N_{c r}$ the equation (12) does not have a solution at all, thus the chiral symmetry is kept unbroken.

The dependence of the critical number $N_{c r}$ on the value of the coupling constant $\alpha$ takes the form

$$
\begin{aligned}
N_{c r}(\alpha) & =\sqrt{\frac{3 \pi}{2 \exp \left(\gamma_{\mathrm{E}}+1\right)}} \frac{1}{\sqrt{\alpha}}-\frac{3}{4} \\
& +\frac{9}{16} \sqrt{\frac{3 \exp \left(\gamma_{\mathrm{E}}+1\right)}{2 \pi}} \sqrt{\alpha}+O(\alpha) .
\end{aligned}
$$

It is quite remarkable that the doublet splitting of the fermion dynamical mass can be rather large. For example, for $\alpha=0.1$ and $N=1$ the mass difference is of 15 orders of magnitude. If one considers for the purposes 


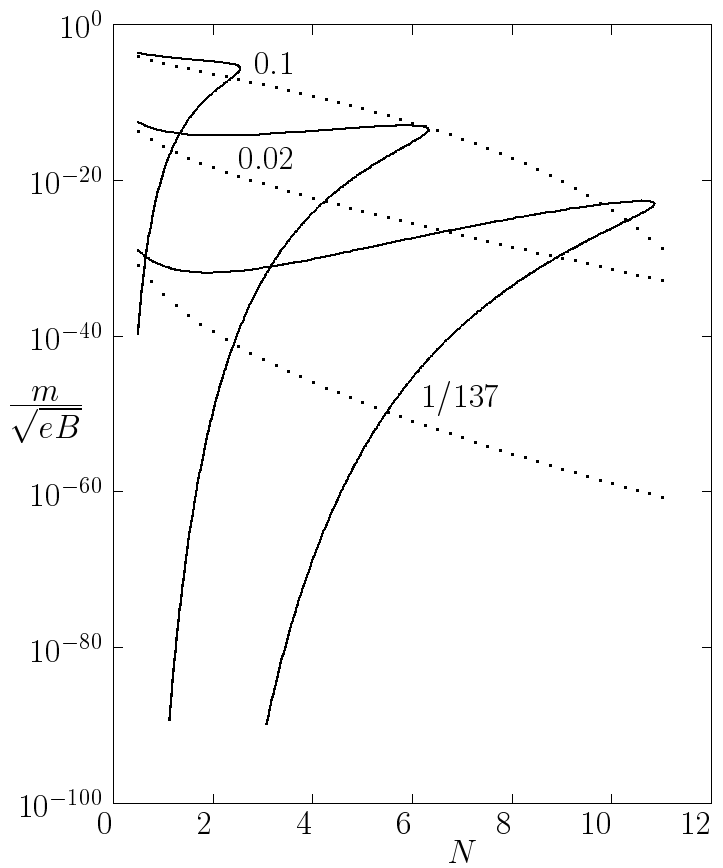

FIG. 3: The dependence of the fermion dynamical mass $m$ divided by $\sqrt{e B}$ on the number of fermions $N$ for different values of the field-free coupling constant, $\alpha=0.1,0.02,1 / 137$. Solid lines correspond to our result Eq. (12). The dotted lines present the results of Refs. 7,8 .

of illustration the magnetic field value $\sim 10^{33} \mathrm{G}$ [16], the two values of the fermion dynamical mass are $\sim 10^{2} \mathrm{GeV}$ and $\sim 10^{-4} \mathrm{eV}$.

We believe that the doublet splitting of the fermion dynamical mass and the conservation of the chiral symmetry at $N>N_{c r}$ as well are new interesting physical phenomena in QED in strong external magnetic field.

We are grateful to V.A. Rubakov and M. V. Chistyakov for helpful discussions.

This work was supported in part by the Russian Foundation for Basic Research under the Grant No. 01-0217334 and by the Ministry of Education of Russian Federation under the Grant No. E00-11.0-5.

* Electronic address: avkuzn@univar.ac.ru

$\dagger$ Electronic address: mikheev@uniyar.ac.ru

[1] B. Jancovici, Phys. Rev. 187, 2275 (1969).

[2] Y. M. Loskutov and V. V. Skobelev, Theor. Math. Phys. 38, 130 (1979).

[3] Y. M. Loskutov and V. V. Skobelev, Theor. Math. Phys. 48, 594 (1982).

[4] V. P. Gusynin and A. V. Smilga, Phys. Lett. B 450, 267 (1999).

[5] Y. M. Loskutov and V. V. Skobelev, Vestn. Mosk. Univ. Fiz. Astron. (in Russian) 24, 95 (1983).

[6] A. V. Kuznetsov, N. V. Mikheev, and M. V. Osipov, Mod. Phys. Lett. A 17, 231 (2002).

[7] V. P. Gusynin, V. A. Miransky, and I. A. Shovkovy, Phys. Rev. Lett. 83, 1291 (1999).

[8] V. P. Gusynin, V. A. Miransky, and I. A. Shovkovy, Nucl. Phys. B 563, 361 (1999).

[9] J. Alexandre, K. Farakos, and G. Koutsoumbas, Phys. Rev. D 62, 105017 (2000).

[10] J. Alexandre, K. Farakos, and G. Koutsoumbas, Phys. Rev. D 64, 067702 (2001).

[11] Y. M. Loskutov and V. V. Skobelev, Vestn. Mosk. Univ. Fiz. Astron. (in Russian) 25, 70 (1984).

[12] Y. M. Loskutov and V. V. Skobelev, Phys. Lett. A 56, 151 (1976).

[13] S. L. Adler, Ann. Phys. N.Y. 67, 599 (1971).

[14] W.-Y. Tsai, Phys. Rev. D 10, 2699 (1974).

[15] A. E. Shabad, Polarization of the vacuum and a quantum relativistic gas in an external field (Nova Sci. Publ., New York, USA, 1992), (Proceedings of the Lebedev Physics Institute, 191).

[16] J. Ambjørn and P. Olesen (1993), hep-ph/9304220. 